\title{
Characterization of Low Molecular Weight Alkoxylated Polymers Using Long Column SFC/MS and an Image Analysis Based Quantitation Approach
}

\author{
J. David Pinkston, Suresh B. Marapane, Glenn T. Jordan, and \\ B. David Clair \\ Miami Valley Laboratories, The Procter and Gamble Company, Cincinnati, Ohio, USA
}

\begin{abstract}
The utility of low viscosity mobile phases and long chromatographic columns for complex polymer analysis is demonstrated. We use long column supercritical fluid chromatography/ mass spectrometry (SFC/MS) with electrospray ionization (ESI) to characterize a variety of complex, low molecular weight polymers. When quantitative analysis is desired, the resulting three-dimensional (time, intensity, and mass-to-charge ratio $[\mathrm{m} / \mathrm{z}]$ ) data are converted to images. Custom image analysis software is used to detect and integrate peaks in arbitrarily defined regions of the time- $\mathrm{m} / \mathrm{z}$ map. These integrated peak volumes can be used to quantitate distinct component classes of the polymer mixtures. (J Am Soc Mass Spectrom 2002, 13, 1195-1208) ( 2002 American Society for Mass Spectrometry
\end{abstract}

\section{$\mathrm{L}$} ow molecular weight (MW) commercial polymers are found in many consumer and industrial tion to an oligomeric or polymeric distribution, they may contain various blocks, capping agents, initiators, additives, and preservatives. Each of these components may itself consist of a distribution of species and chain lengths. Relatively small differences in composition or structure can at times greatly impact performance [1]. Numerous analytical methods can be used to help understand these differences, including nuclear magnetic resonance spectroscopy, infrared spectroscopy, and chromatographic methods such as gel-permeation chromatography. Advances in ionization methods, such as matrix-assisted laser desorption/ionization, electrospray ionization, and potassium ion desorption/ionization, and in mass analyzers such as time-of-flight mass spectrometers, have brought mass spectrometry firmly into the fold of methods used to study polymeric and oligomeric mixtures [2-4].

As in the analysis of any complex mixture, the combination of a high efficiency separation method with an informative spectroscopic method is a powerful tool. Packed column supercritical fluid, subcritical fluid, and enhanced fluidity liquid chromatographies have been used to separate low-MW polymeric and oligomeric mixtures in the past [5-8]. While these three

Published online September 6, 2002

Address reprint requests to Dr. J. D. Pinkston, Miami Valley Laboratories, The Procter and Gamble Company, P.O. Box 538707, Cincinnati, OH 45253-8707, USA. E-mail: pinkston.jd@pg.com techniques are different, they are closely related and require the same instrumentation. All three will be referred to as SFC in this report. The addition of a significant amount, typically 20 to $95 \%$, of a viscositylowering agent, such as carbon dioxide, to the mobile phase provides a number of advantages for low-MW polymer separations [9]. Lower viscosity allows the use of higher mobile phase flow rates (i.e., faster analyses [10]) and/or longer columns (i.e., higher efficiencies [11]) than when using traditional liquid mobile phases. Compared to liquid chromatography, these higher mobile phase flow rates can often be attained without a concomitant loss in chromatographic efficiency since diffusion coefficients and optimum velocities are typically higher with SFC. While $\mathrm{CO}_{2}$ itself is relatively nonpolar, mixtures of $\mathrm{CO}_{2}$ and polar organic solvents retain the polarity and solvating power of the polar organic until significant levels $(50-60 \%)$ of $\mathrm{CO}_{2}$ are added [12]. These mixtures can often be used to separate all but the most polar, poly-ionic polymers.

Mass spectrometry (MS) is arguably the most widely used informative detector in chromatography. Interfacing SFC to mass spectrometry using an atmosphericpressure-ionization source is relatively straightforward and has been described previously [13-15]. The selfnebulizing nature of a mobile phase consisting of $\mathrm{CO}_{2}$ and an organic "modifier" results in improved response compared to conventional LC/MS [16]. Pneumatically assisted electrospray ionization provides simple spectra usually consisting of a protonated molecule and a sodium or ammonium (when volatile ammonium salts are added) adduct ion, or both, in the positive mode. 
The predictable nature of these spectra simplifies data interpretation when simple mixtures are present in the ion source, as occurs when analytes coelute in a chromatographic separation.

The data resulting from a chromatography/MS separation is three dimensional in nature $(\mathrm{m} / \mathrm{z}$, retention time, and intensity). The third dimension provided by the mass spectrometer, $m / z$, has the practical effect of enhancing the resolving power (in the sense of information per unit time) of the chromatographic system. Many commercial chromatography/MS data systems provide the ability to generate 2-D representations of these 3-D data, called contour plots, or maps. However, the ability to manipulate and analyze these 2-D representations is often limited. The contour plots resulting from long column SFC/MS of complex polymers often contained many hundred, sometimes thousands, of peaks. We found the contour plot a very efficient means of viewing the resulting data, but sought more extensive data analysis possibilities tied to the contour plots.

We therefore developed software that converts the SFC/MS data to an image, which can be viewed, manipulated, and analyzed with commercially available image analysis software. Furthermore, the software we developed allows the analyst to define arbitrary regions within the image, integrate peaks within this region, and export the results to a widely used commercial spreadsheet program.

In this report we demonstrate the use of long packed columns, high mobile phase flow rates, and MS detection with pneumatically-assisted electrospray ionization for efficient SFC separations and qualitative analysis of complex low-MW polymers. We also describe the development of image analysis tools for the quantitative analysis of chromatography/MS data.

\section{Experimental}

\section{Safety Considerations}

The instrumentation used in this work operated at relatively high pressures and high temperatures. Pressures were similar to those used in traditional high performance liquid chromatography. However, temperatures were often significantly higher than those used in HPLC. Correspondingly, researchers must be aware of both the pressure and temperature limits of columns and other instrumental components before attempting to reproduce this work.

\section{Chemicals and Mobile Phase Components}

Ammonium acetate and methanol (HPLC Grade) for preparation of the mobile phase, the pressure regulating fluid, and of the sheath-flow liquid were purchased from J. T. Baker (Phillipsburg, NJ). Carbon dioxide was SFC/SFE grade obtained from Air Products and Chemicals, Inc. (Allentown, PA). Distilled-deionized water was provided by a Milli-Q Water Purification System
(Millipore Corp., Bedford, MA). Toluene (ACS Reagent Grade) for sample dissolution was obtained from J. T. Baker.

\section{Sample Preparation}

Purified (single component) deca(oxyethylene)octanol $\left(\mathrm{C}_{8} \mathrm{E}_{10}\right)$ was used as an internal standard. The $\mathrm{C}_{8} \mathrm{E}_{10}$ and most of the low-MW polymer samples were prepared within the laboratories of The Procter and Gamble Company. Most of these polymers were of the multiblock "A-B-C" variety, where A, B, and C consisted of either fatty alkyl, poly(ethylene glycol), or poly(propylene glycol) chains of various lengths. Other low-MW polymer samples were obtained from a variety of commercial sources. Neodol 25-12 $\left(\mathrm{C}_{12}, \mathrm{C}_{13}, \mathrm{C}_{14}\right.$, and $C_{15}$ alcohols ethoxylated to an average ethoxylate chain length of 12) was obtained from Shell Chemical (Houston, TX). Solutions of low-MW polymer samples were prepared at approximately $0.05 \mathrm{mg} / \mathrm{mL}$ in toluene. A stock solution of the $\mathrm{C}_{8} \mathrm{E}_{10}$ internal standard was prepared in toluene at a concentration of $1.01 \mathrm{mg} / \mathrm{mL}$. An aliquot of the $\mathrm{C}_{8} \mathrm{E}_{10}$ internal standard stock solution was added to each sample such that the final concentration of internal standard was $5.05 \mu \mathrm{g} / \mathrm{mL}$. These solutions were injected into the SFC system without further processing.

\section{SFC/MS Instrumentation}

The SFC system was a Model G1205A (Hewlett-Packard, now Agilent, Wilmington, DE). This instrument was upgraded with a Model FCM-1200 fluid control module, a Model 719 autosampler, and Berger Instruments SFC ChemStation control software, version 3.3.9 (all from Berger Instruments, Newark, DE). The chromatographic column consisted of one, three, four, or eight $4.6 \mathrm{~mm}$ i.d., $250 \mathrm{~mm}$ long, $4.6 \mathrm{~mm}$ i.d. DeltaBond Cyano columns (Thermo Hypersil, Bellefonte, PA) coupled in series for a total length of $0.25,0.75,1$, or $2 \mathrm{~m}$, respectively. Unless otherwise indicated, the "standard" conditions consisted of a $1 \mathrm{~m}$ column, a flow rate of $2 \mathrm{~mL} / \mathrm{min}$ (measured at the pumps), a post-column pressure of 200 bar, and a column oven temperature of $100{ }^{\circ} \mathrm{C}$. The mobile phase composition was held at $3 \%$ methanol in $\mathrm{CO}_{2}$ for 3 min upon injection, then was raised to $30 \%$ methanol at a rate of $0.5 \% / \mathrm{min}$, where it was held until the end of the separation. The injection volume was $10 \mu \mathrm{L}$.

Post-column pressure control is provided by the pressure regulating fluid interface, as described previously $[15,17]$. Briefly, a low dead volume chromatographic tee is located between the UV absorbance detector and the electrospray interface. A fluid, here methanol, is pumped into the one arm of the tee under pressure control using a Model D Series 260 syringe pump (Isco, Lincoln, NE). The chromatographic effluent enters a second arm, and the resulting mixture exits the tee and flows to the mass spectrometer. The tee was 

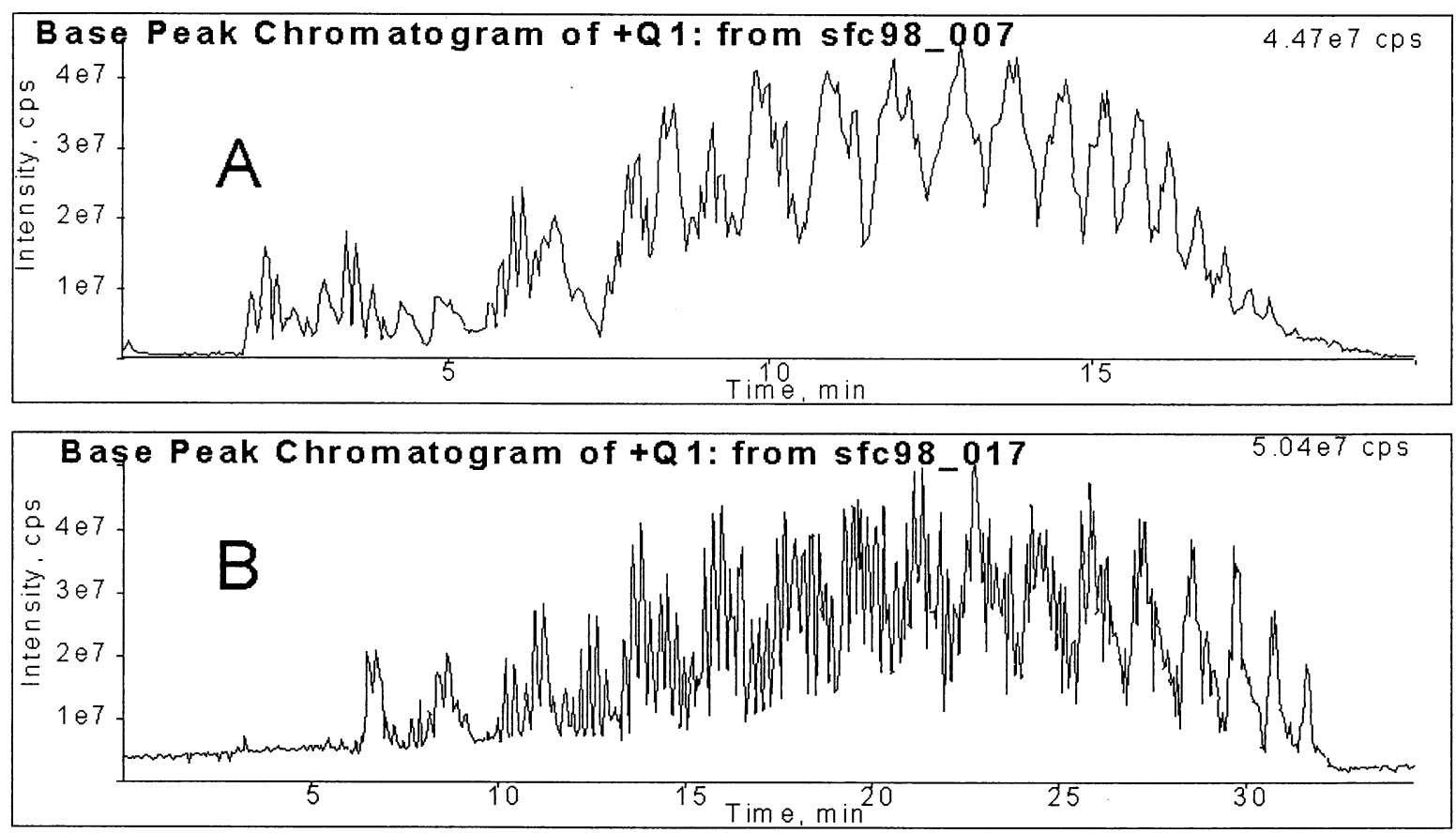

Figure 1. Separations of a low-MW alkoxylated polymer using column lengths of (a) $25 \mathrm{~cm}$, and (b) $75 \mathrm{~cm}$. Outlet pressure $(200 \mathrm{bar})$, column temperature $\left(100{ }^{\circ} \mathrm{C}\right)$, and flow rate $(2 \mathrm{~mL} / \mathrm{min})$ were identical for both runs. The average modifier gradient rate was reduced from $1.59 \% / \mathrm{min}$ for the $25 \mathrm{~cm}$ column to $0.5 \% / \mathrm{min}$ for the $75 \mathrm{~cm}$ column to take advantage of its full length.

located near the TurboIonspray source. A $75 \mu \mathrm{m}$ i.d., $195 \mu \mathrm{m}$ o.d., $30 \mathrm{~cm}$ length of deactivated fused silica tubing (Dionex, Sunnyvale, CA) carried the effluent from the tee, through the TurboIonspray interface probe, to the union within the probe, located just before the sprayer tip. The original PEEK tubing within the probe was replaced with $1 / 16$ inch o.d., 0.020 inch i.d. PEEK tubing to accommodate the fused silica transfer line. The interface provides pressure regulation with good chromatographic fidelity, as well as additional methanol for efficient electrospray ionization when the modifier (i.e., methanol) content of the mobile phase is low. The pressure regulating fluid flow typically ranged from 0.3 to $0.8 \mathrm{~mL} / \mathrm{min}$.

As described previously $[15,17]$, a sheath-flow liquid of 80:20 methanol/water containing $1 \mathrm{mM}$ ammonium acetate flowed within the PEEK tubing in the Turbolonspray probe, concentric to the fused silica transfer line, to the union located just before the sprayer tip. The effluent and the sheath-flow liquid mixed within the union and flowed through the sprayer tip. The sheathflow liquid was delivered by a second Model D Series 260 syringe pump and provided enhanced ionization $[15,17]$.

The mass spectrometer was a PE Sciex API-365 (PE Sciex, Thornhill, Ontario, Canada). The Turbolonspray source was used with a Turbo gas flow of $8 \mathrm{~L} / \mathrm{min}$ at a nominal temperature of $450{ }^{\circ} \mathrm{C}$. The nebulizer gas pressure was 60 psi (nitrogen).

The ESI, orifice, and multiplier potentials were held at 5000, 50, and $-2000 \mathrm{~V}$, respectively. Q1 was scanned from $\mathrm{m} / \mathrm{z} 200$ to $\mathrm{m} / \mathrm{z} 2000$ in "peak hopping" mode with a step value of $1.0 \mathrm{u}$, a $1.0 \mathrm{~ms}$ dwell time, a $5 \mathrm{~ms}$ interscan pause, and a 55 millimass units $(\mathrm{mmu}) / 100 \mathrm{u}$ correction factor (discussed below). Q2 and Q3 were operated in rf-only mode. The mass spectrometer was operated in peak hopping mode to allow scanning over the $1800 \mathrm{~m} / \mathrm{z}$ range in approximately $2 \mathrm{~s}$. Nevertheless, the scan cycle time of $2 \mathrm{~s}$ only allowed the acquisition of between 5 and 11 scans per peak because of the narrow widths of the SFC peaks throughout the chromatogram. This scan cycle time limitation prevented the use of higher flow rates $(3-4 \mathrm{~mL} / \mathrm{min})$, which were entirely reasonable from a chromatographic point of view, and which would have reduced the total analysis time. Higher flow rates would have resulted in peak widths too narrow to adequately sample with the mass spectrometer used in this work. While peak hopping provides a sufficiently rapid scan speed, it forces the analyst to choose an expected atomic composition for the analytes. We knew that the polymers we studied were primarily composed of carbon, hydrogen, oxygen, and nitrogen, which dictated a correction factor of 55 $\mathrm{mmu} / 100 \mathrm{u}$. Molecules containing other atoms, such as halogens, might not be detected because of the peak hopping mode of acquisition. Also, other important information that can usually be derived from mass spectra, such as isotope ratios, is not acquired in peak hopping mode. One obvious alternative in this situation is to use a mass spectrometer with a faster acquisition rate, such as one of the newer vintage quadrupole mass spectrometers, where hardware and software changes 


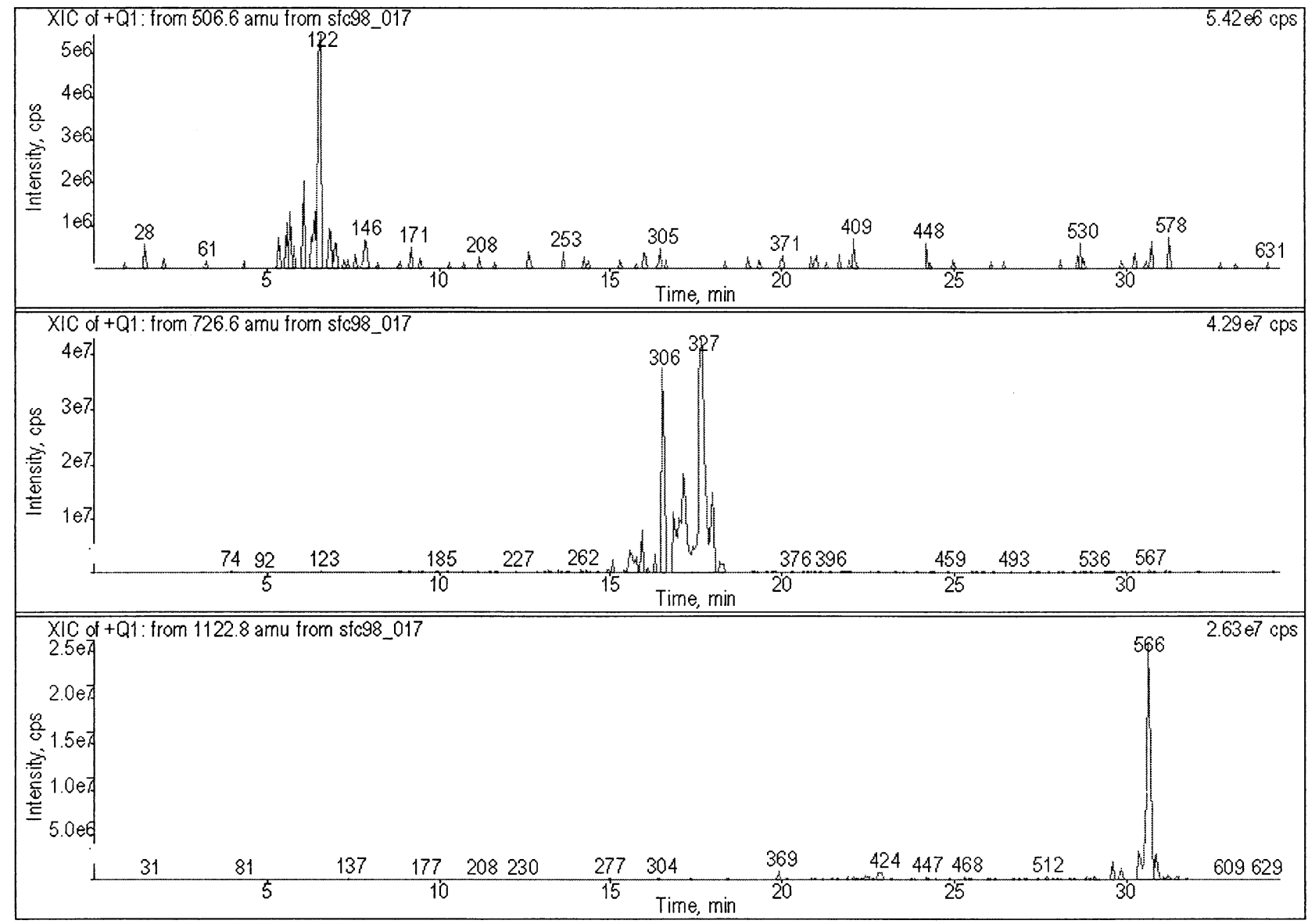

Figure 2. Mass chromatograms of three members of an ethoxylated oligomeric series extracted from the lower chromatogram shown in Figure 1. The component of interest in the middle mass chromatogram is the peak eluting at scan number 327.

allow faster scan rates without resorting to peak hoping, or, even better, a time-of-flight mass spectrometer. Unfortunately, none of these was available to us for the work described herein.

\section{Image Analysis}

We developed a novel image analysis based technique to provide the ability to visualize the SFC/MS data set as an image with columns being time, rows being $\mathrm{m} / \mathrm{z}$, and brightness (color) being mass spectral intensity. The SFC/MS data file is first converted to Matlab (The Mathworks, Inc., Natick, MA) format using a script program provided by PE-Sciex. Our custom software allows the analyst to (1) import the Matlab-format data file and convert this file to an image data format; (2) remove the majority of mass spectrometric background from the data in an automated fashion; (3) define arbitrary regions of interest within the image using a graphical user interface pointing device (a mouse); (4) define integration threshold levels and other integration parameters; (5) detect and integrate peaks within the region; and (6) export the results to a Microsoft Excel spreadsheet. This procedure is implemented in the
Optimas image analysis package (version 6.5.171, Media Cybernetics, L.P., Silver Spring, MD) using custom macros. Readers may contact the corresponding author to obtain copies of the macros. The background removal process is simple, but merits description. First, the image is divided into the minimum number of time slices that will provide adequate background removal. This minimum number will vary depending on the rate of change of the background and on the peak widths of the analytes. If the background level varies in such a way that three or four distinct regions are observed by the analyst, which is typical for a 50 min separation, the number of time slices is three or four, respectively. The signal at each $\mathrm{m} / \mathrm{z}$ is then averaged over the time slice. Finally, this average is subtracted from the signal to produce a background subtracted signal. As long as the background is relatively constant over the time slice, the peak width of each analyte peak is narrow with respect to the time slice, and the intensity contribution of analyte peaks to the average signal is small (5\% or less) (i.e., the peaks at each $\mathrm{m} / \mathrm{z}$ are sparse); this simple approach provides adequate background removal. These conditions have been satisfied in all the SFC/MS data sets examined to date. 


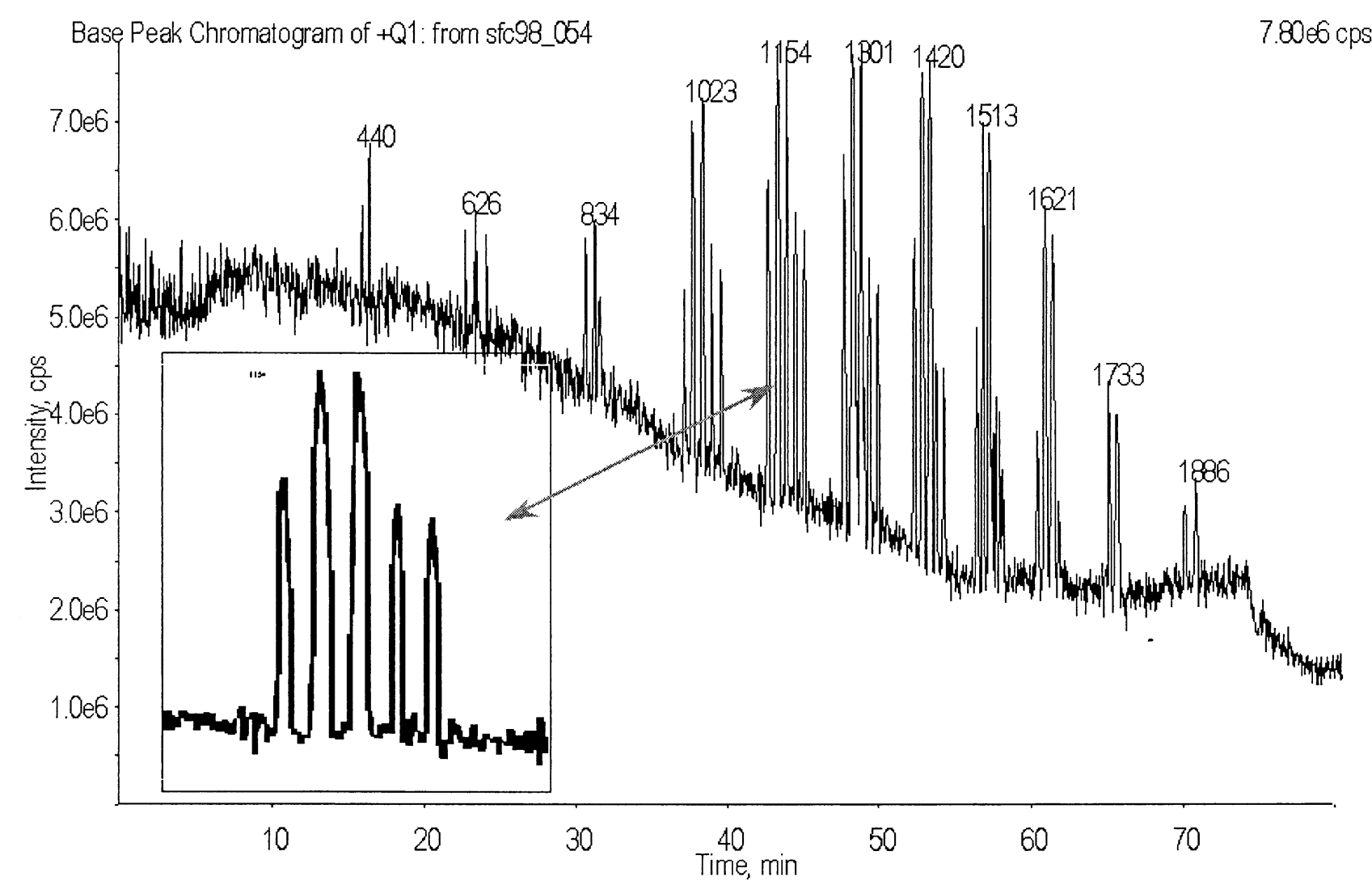

Figure 3. Separations of the same low-MW alkoxylated polymer as shown in Figure 1 using a column length of $2 \mathrm{~m}$. The numbers above the peaks are scan numbers. See the Results and Discussion section for details.

\section{Results and Discussion}

\section{Effect of Column Length}

The benefits of using long packed columns in the SFC analysis of pharmaceuticals and compounds of environmental interest have been previously demonstrated [11]. Figure 1 illustrates the impact of column length on the SFC characterization of low-MW polymers. The upper portion of the figure shows the base peak chromatogram of a low-MW polymer mixture of the A-B-C variety (see Experimental section) using a column with a length of $25 \mathrm{~cm}$, while the lower portion of the figure shows the corresponding separation using a $75 \mathrm{~cm}$ column. The column i.d. $(4.6 \mathrm{~mm})$, the outlet pressure (200 bar), the flow rate $(2 \mathrm{~mL} / \mathrm{min})$, and the column temperature $\left(100^{\circ} \mathrm{C}\right)$ were identical for both separations. The mobile phase gradient began at 3\% methanol in $\mathrm{CO}_{2}$ and ended at $30 \%$, but the gradient rate was altered from an average rate of $1.59 \% / \mathrm{min}$ for the $25 \mathrm{~cm}$ column to $0.5 \% / \mathrm{min}$ for the $75 \mathrm{~cm}$ column to make effective use of the longer column. (Maintaining a gradient rate of $1.59 \% / \mathrm{min}$ with the longer column would have resulted in the analytes achieving an effective $k^{\prime}$ of zero [i.e., no retention] long before their elution. The remainder of the long column would have simply operated as an empty transfer tube, in which only peak broadening and no chromatographic separa- tion was taking place, from that point forward. The slower gradient makes full use of the entire column.) A base peak chromatogram is obtained by plotting the intensity of the base peak in each spectrum versus retention time. The base peak chromatogram is useful in comparing separations such as those in Figure 1 because it eliminates background and highlights major peaks. While the lower chromatogram may simply appear "noisier" than the upper at first glance, closer inspection of the data reveals that the merged multiplets in the upper chromatogram are resolved into many individual peaks in the lower chromatogram.

Figure 1 illustrates the improved chromatographic resolution provided by a longer column. Beyond a simple visual comparison, the peak capacity, $\mathrm{n}_{\mathrm{c}}$, is a useful tool for comparing the separating power of chromatographic systems, even in gradient mode operation (where parameters such as chromatographic efficiency cannot be calculated) [18]. The peak capacity is a measure of the number of peaks that may be resolved, at a specified resolution, by a chromatographic system within a given period of time or distance. In order to compare two systems, the unit of time or distance used must be comparable. It has often been used in recent years to compare the separating power of chromatographic systems during the period of time of a mobile phase gradient. A resolution of 1.0 has often been used, 


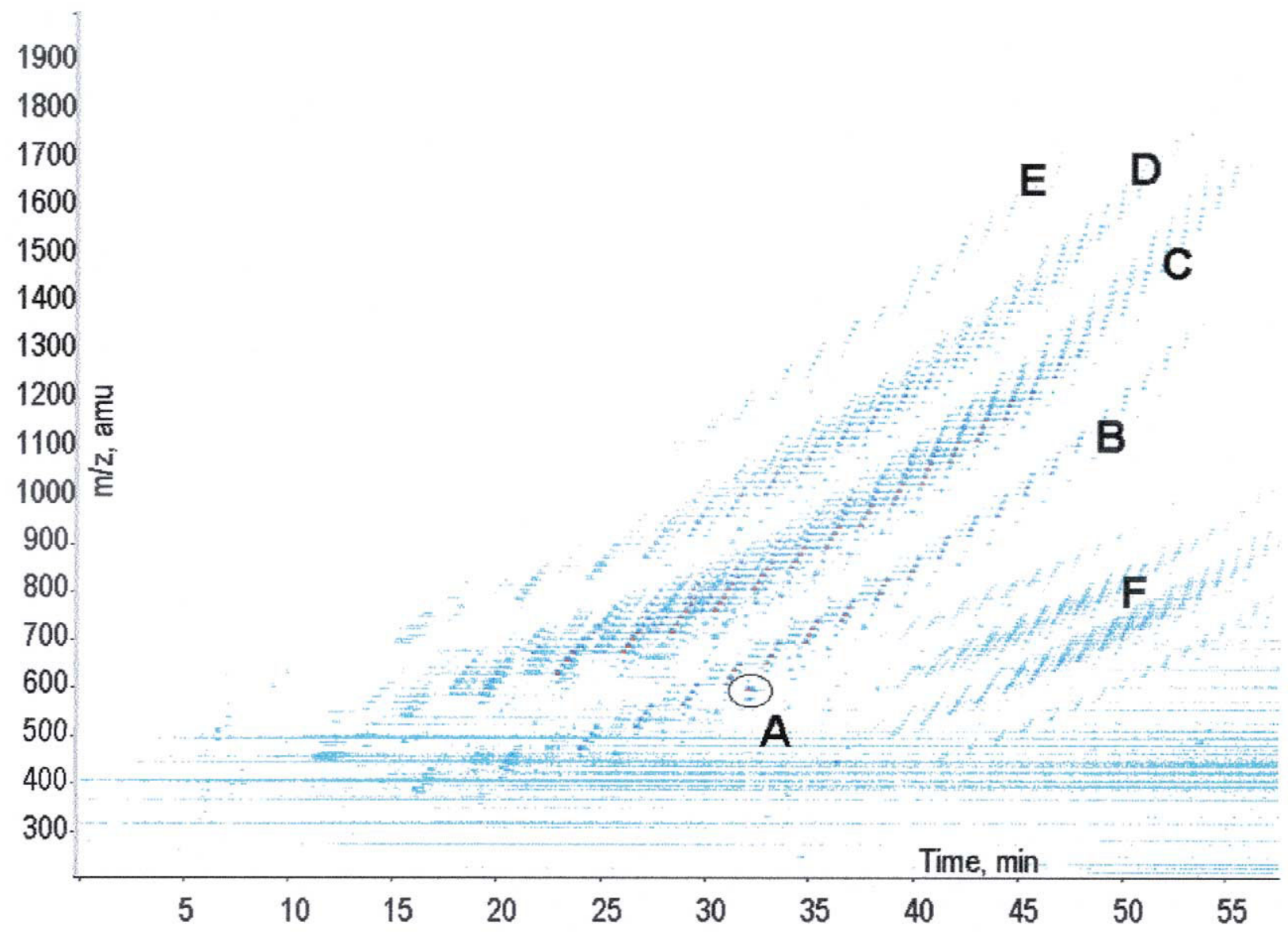

Figure 4. Contour plot of a low-MW alkoxylated polymer. (A) C8E10 (single component) internal standard; (B) unreacted starting material with an alkyl capping group on only one end of the molecule; (C) reaction product with alkyl capping groups on both ends of the molecule; (D) reaction product with a dimerized alkyl capping group on one end of the molecule; (E) reaction product with a trimerized alkyl capping group on one end of the molecule; (F) doubly-charged species.

though this may not be appropriate for some applications [18]. By inspecting mass chromatograms of individual components to obtain an average peak width, one may estimate $\mathrm{n}_{\mathrm{c}}$ for the chromatograms in Figure 1 from the beginning to the end of the gradient. At a resolution of $1.0, n_{c}$ for the $25 \mathrm{~cm}$ system is 36 , while $n_{c}$ for the $75 \mathrm{~cm}$ system is 216 . Of course, the improved separation comes at the expense of time. The separation using the longer columns requires approximately 35 min, roughly $75 \%$ longer than the separation using the $25 \mathrm{~cm}$ column. Figure 1 also illustrates another aspect of gradient SFC separations-with respect to the total analysis time, the peak widths are relatively narrow throughout a separation. The chromatographic peaks for the ammonium adduct ions of three members of a homologous, oligomeric series eluting over the course of the separation conducted with the $0.75 \mathrm{~cm}$ column have peak widths at half height of $0.20 \mathrm{~min}$ (at an elution time of $6.6 \mathrm{~min}$, with an ethoxylate chain length of 2), $0.20 \mathrm{~min}$ (at $17.7 \mathrm{~min}$, with an ethoxylate chain length of 7), and $0.12 \mathrm{~min}$ (at $30.6 \mathrm{~min}$, with an ethoxylate chain length of 16). Mass chromatograms for the ammonium adduct ions of these three oligomers are shown in Figure 2. Further experiments showed that separations with mobile phase flow rates of 3 and 4 $\mathrm{mL} / \mathrm{min}$ provided $\mathrm{n}_{\mathrm{c}} \mathrm{s}$ nearly as high as those measured with $2 \mathrm{~mL} / \mathrm{min}$ flow rates, but with shorter analysis times and narrower peaks. As described in the Experimental section, we are not able to use such flow rates because of the scan time limitations of the quadrupole mass spectrometer used in this work.

As one might expect, a longer column provides even better resolution for low-MW polymers. As the column length is increased, the inlet pressure and the total analysis time also increase. Eventually, the inlet (i.e., pumping system) pressure limit is reached, and the analysis time becomes impractically long. Figure 3 illustrates a separation of the same polymeric mixture featured in Figure 1 near the practical limit of column length for this system, $2 \mathrm{~m}$. This separation cannot be compared directly to those in Figure 1 because a number of conditions were altered to improve peak resolution and to reduce the total analysis time. While Figures 1 and 3 cannot be directly compared, Figure 3 illustrates what is possible in the extreme. The column temperature was increased to $140{ }^{\circ} \mathrm{C}$, the initial methanol con- 


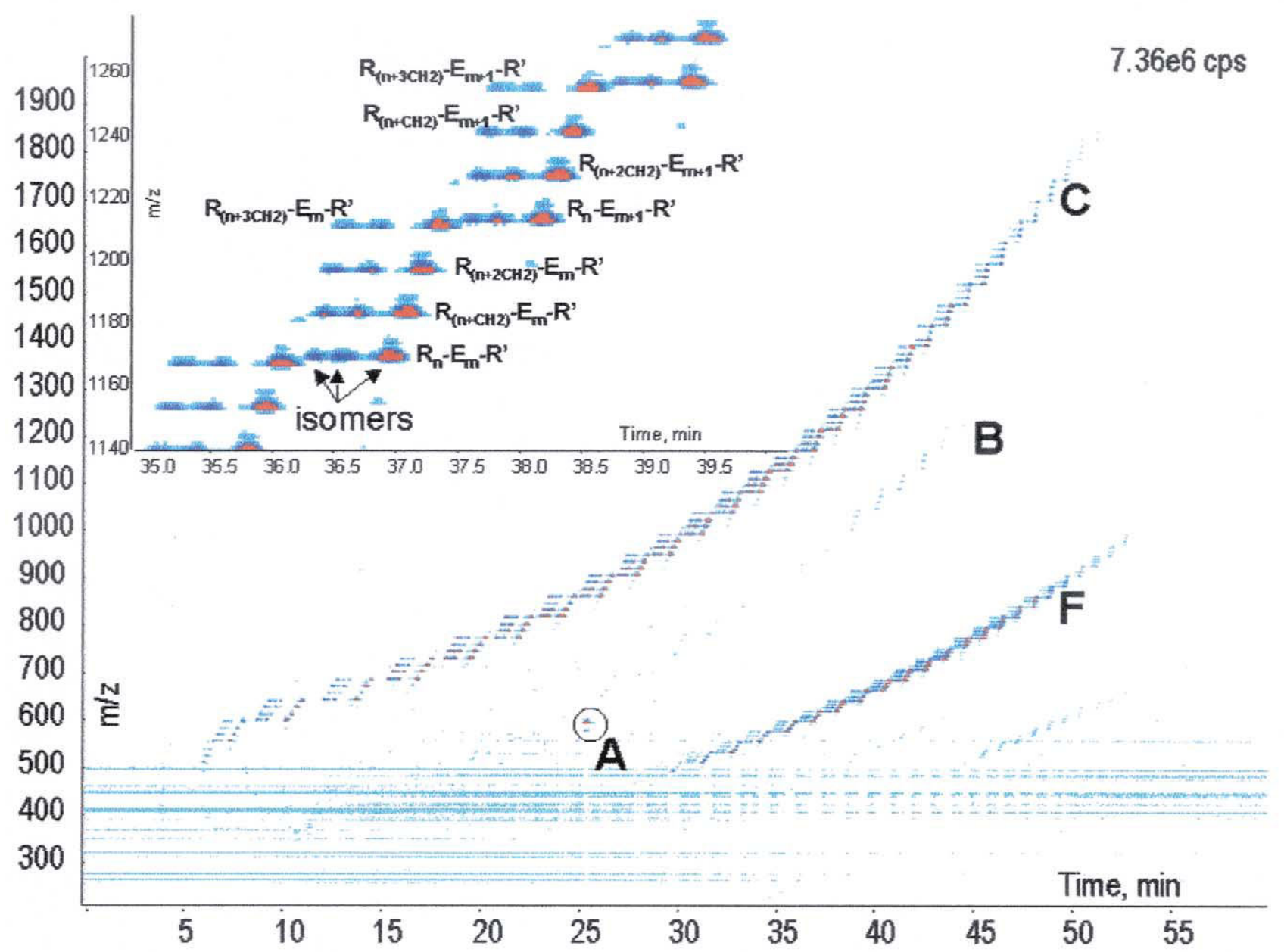

Figure 5. Contour plot of a low-MW alkoxylated polymer similar to that shown in Figure 4, but synthesized via a different pathway. Ion series labels are the same as those used in Figure 4. See the Results and Discussion section for a description of the inset.

tent in the mobile phase was $15 \%$, the final methanol content in the mobile phase was $40 \%$, and the mobile phase composition gradient rate was reduced to $0.3 \% /$ $\mathrm{min}$. The mass spectrometric scan range was from $\mathrm{m} / \mathrm{z}$ 200-1100 rather than from $m / z 200-1500$. The estimated $\mathrm{n}_{\mathrm{c}}$ for this separation is 237 . However, the total analysis time is approximately $80 \mathrm{~min}$.

We chose a column length of $1 \mathrm{~m}$, a column temperature of $100{ }^{\circ} \mathrm{C}$, a mobile phase gradient of 3 to $30 \%$ methanol in $\mathrm{CO}_{2}$ at $0.5 \% / \mathrm{min}$, a flow rate of $2 \mathrm{~mL} / \mathrm{min}$, and a post-column pressure of $200 \mathrm{bar}$ as a practical compromise between peak resolution, analysis time, peak width, and method durability. Most of the separations of low-MW polymers performed with these conditions were completed in less than $1 \mathrm{~h}$. Except as noted, these were the conditions used for the separations described in the following sections.

\section{Examples of Low-MW Polymer Separations}

As mentioned in the introduction, we found contour plot chromatograms (2-D representations of 3-D
SFC/MS data) very useful in examining and comparing the separations of low-MW polymers. The contour plot is a compilation of hundreds of mass spectra. A vertical slice taken from the contour plot would represent a single traditional mass spectrum, as illustrated later in this work. Figure 4 shows the contour plot of the low-MW polymer very similar in nature to the polymer featured in the base peak chromatograms of Figures 1 and 3. This polymer essentially consists of a poly(oxyethylene) (a.k.a. polyethyleneglycol, or ethoxylate) chain capped by an alkyl chain on each end. Additional complexity is introduced by the fact that a variety of alkyl chain lengths are present, that the alkyl chains are present in a variety of isomers (that are chromatographically resolved, with branched isomers eluting before straight chain isomers [19]), that the capping alkyl chains are sometimes present as dimers, trimers, etc., and that unreacted starting material and other minor reaction byproducts are also present. Each species is represented by an ammonium adduct ion and a minor sodium adduct ion, with a very small protonated molecule. The ammonium and sodium adduct ions are 


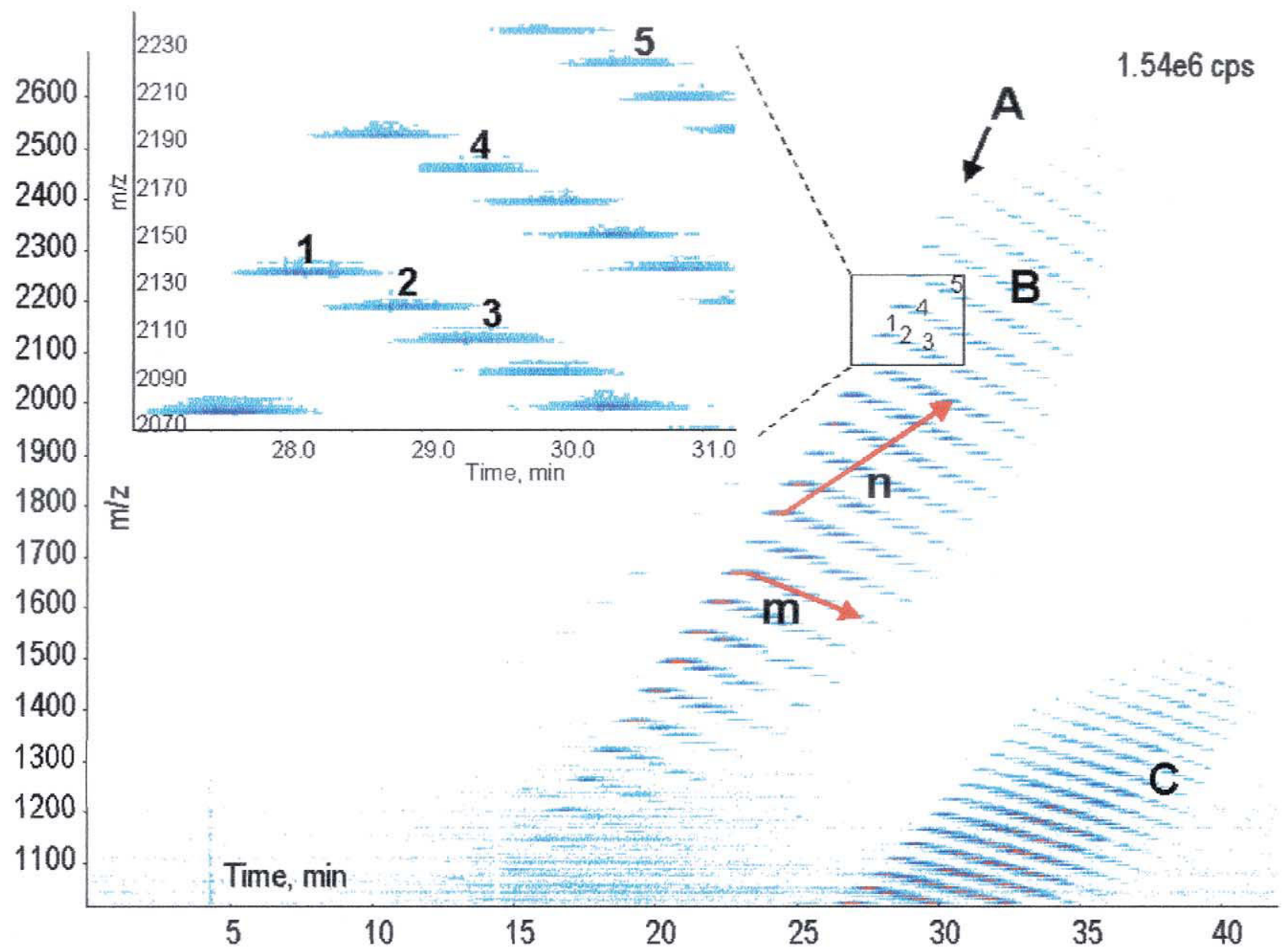

Figure 6. Contour plot of a commercial EO-PO polymer (a mixed ethoxylate-propoxylate polymer). (A) poly(propylene glycol) (PPG) containing no ethoxylate groups; (B) mixed EO-PO polymers; (C): doubly-charged species. Arrow $n$ indicates the progression of peaks in which successive ethoxylate groups are added to the PPG polymer. Arrow $\mathrm{m}$ indicates the progression of peaks in which ethoxylate groups are substituted for propoxylate groups in the polymer. The enclosed region is expanded, and the peak numbers correspond to those in Table 1.

separated by only $5 \mathrm{~m} / \mathrm{z}$ units, and appear essentially as one spot on the scale of the contour plot in Figure 4. Thus, each component is essentially represented by a single spot. Note that ionization efficiencies of alkoxylated polymers in ESI are known to vary with structure, and are especially poor for alkoxylate chain lengths shorter than two [20]. Thus, as in any mass spectrometric technique, one cannot make a direct inference about the relative amounts of two components with very different structures based solely upon their relative peak areas (or peak volumes in a 3-D representation). While estimates of relative levels of species with similar structures can be performed based on relative peak volumes, calibration with known standards is essential for accurate quantitation.

The main features and series of Figure 4 are the $\mathrm{C}_{8} \mathrm{E}_{10}$ internal standard (Figure 4A), uncapped starting material (essentially a series of ethoxylated alcohols) (Figure $4 \mathrm{~B})$, the desired product (Figure 4C), and reaction products capped with dimeric (Figure 4D), trimeric
(Figure 4E), etc. capping agents. The clusters of singlycharged ions in each series are separated by $m / z 44$, which one would expect for an ethoxylated series. Within each cluster, the species are separated by $m / z 14$, corresponding to one methylene group. The higher MW members of the polymeric series tend to be present as both singly and doubly charged species. The doubly charged ions are present in the lower right quadrant of

Table 1. Most abundant $\mathrm{m} / \mathrm{z}$ and structures for labeled peaks in Figure 6

\begin{tabular}{lcc}
\hline $\begin{array}{l}\text { Peak } \\
\text { number }\end{array}$ & $\begin{array}{c}\text { Most } \\
\text { abundant } \\
m / z\end{array}$ & \multicolumn{1}{c}{ Structure } \\
\hline \hline 1 & 2126 & {$\left[\mathrm{HO}-\left(\mathrm{C}_{3} \mathrm{H}_{6} \mathrm{O}\right)_{36}-\mathrm{H}+\mathrm{NH}_{4}\right]^{+}$} \\
2 & 2112 & {$\left[\mathrm{HO}-\left(\mathrm{C}_{3} \mathrm{H}_{6} \mathrm{O}\right)_{35}-\left(\mathrm{C}_{2} \mathrm{H}_{4} \mathrm{O}\right)-\mathrm{H}+\mathrm{NH}_{4}\right]^{+}$} \\
3 & 2098 & {$\left[\mathrm{HO}-\left(\mathrm{C}_{3} \mathrm{H}_{6} \mathrm{O}\right)_{34}-\left(\mathrm{C}_{2} \mathrm{H}_{4} \mathrm{O}\right)_{2}-\mathrm{H}+\mathrm{NH}_{4}\right]^{+}$} \\
4 & 2170 & {$\left[\mathrm{HO}-\left(\mathrm{C}_{3} \mathrm{H}_{6} \mathrm{O}\right)_{36}-\left(\mathrm{C}_{2} \mathrm{H}_{4} \mathrm{O}\right)-\mathrm{H}+\mathrm{NH}_{4}\right]^{+}$} \\
5 & 2214 & {$\left[\mathrm{HO}-\left(\mathrm{C}_{3} \mathrm{H}_{6} \mathrm{O}\right)_{36}-\left(\mathrm{C}_{2} \mathrm{H}_{4} \mathrm{O}\right)_{2}-\mathrm{H}+\mathrm{NH}_{4}\right]^{+}$} \\
\hline
\end{tabular}




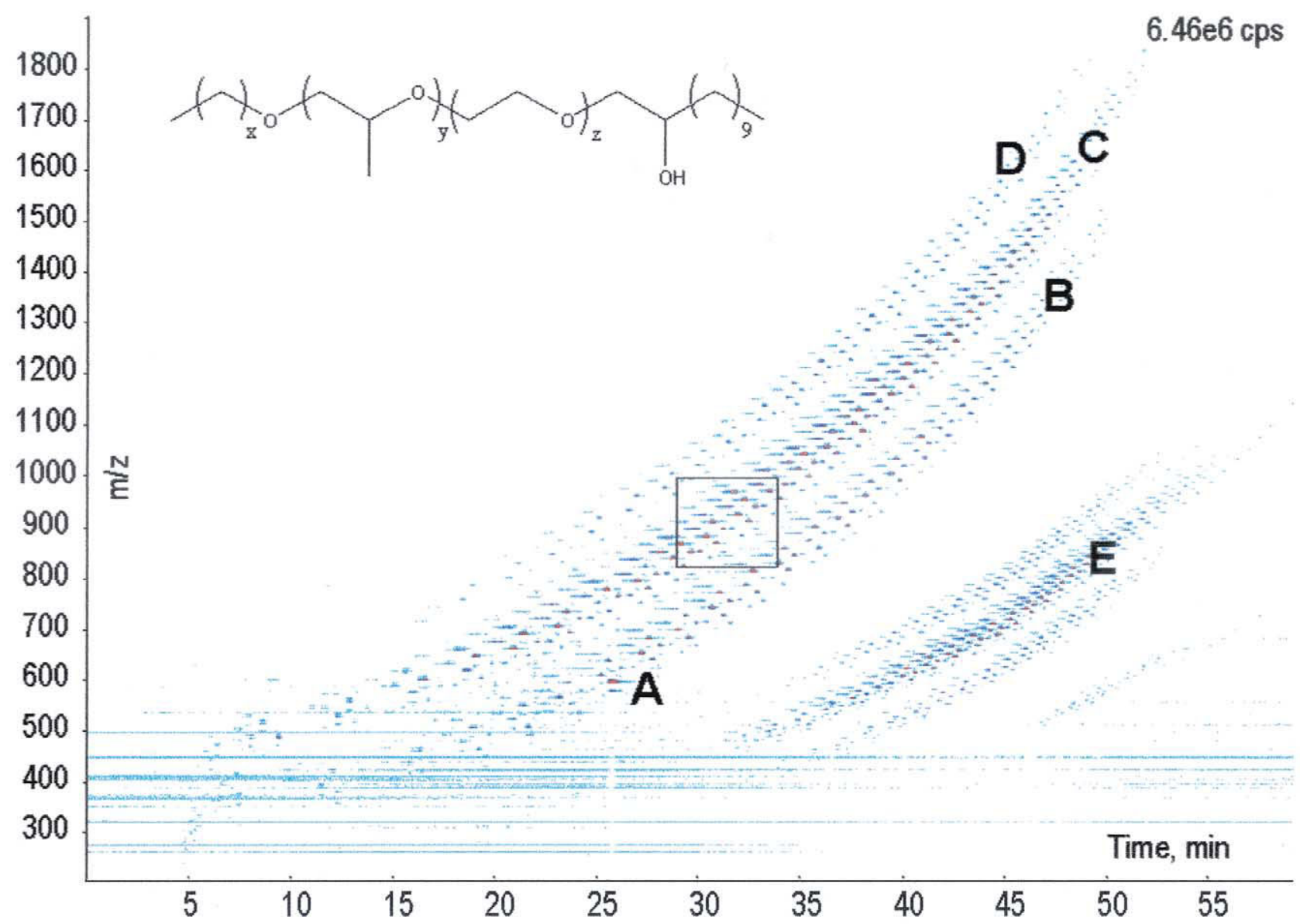

Figure 7. Contour plot of a di-capped EO-PO polymer. (A): C8E10 (single component) internal standard; (B): singly capped EO-PO chain, lacking the $\mathrm{C}_{12}-\mathrm{OH}$ chain; $(\mathbf{C})$ : di-capped EO-PO chain, as shown in the structure; $(\mathbf{D})$ : di-capped EO-PO chain, but the $\mathrm{C}_{12}-\mathrm{OH}$ cap is a dimer $\left(\mathrm{C}_{12}-\mathrm{O}-\mathrm{C}_{12}-\mathrm{OH}\right)$; (E): doubly charged species. $x$ has values of 5,7 , and 9 , y ranges from 0 to 3 , and $z$ from $\sim 3$ to at least 34. The enclosed region is enlarged in Figure 9.

the plot (Figure 4F). Finally, horizontal lines present at low $\mathrm{m} / \mathrm{z}$ are due to background ions derived from solvent clusters, column bleed, etc. which are typical in ESI.

A similar low-MW polymer was synthesized using an alternative synthetic method designed to avoid the formation of dimeric, trimeric, etc. capping groups. The monomeric capping agent did not carry a reactive hydroxyl group that could react with other capping groups during the reaction to form dimeric, trimeric, etc. caps. The contour plot of this mixture is shown in Figure 5. This plot shows a small amount of the residual, unreacted ethoxylated alcohol, the desired mono-capped product, doubly and triply charged ions for the higher MW members of the series, and the $\mathrm{C}_{8} \mathrm{E}_{10}$ internal standard. There are no signs of dimeric- and trimeric-capped reaction products as shown in Figure 4, thus demonstrating that the alternative synthesis was successful. The inset enlargement in Figure 5 shows a portion of the desired product region corresponding to two ethoxylate chain lengths ( $m$ and $m+1)$. From other work, we know that the capping alkyl chain on one end of the ethoxylate chain (R') is of a single chain length and isomer. However, the capping alkyl chain on the other end of the ethoxylate chain $\left(\mathrm{R}_{\mathrm{n}+\mathrm{xCH} 2}\right)$ has a distribution of chain lengths and isomers. There are four primary chain lengths, each differing by a single methylene group $(14 \mathrm{Da})$, providing a distribution of peaks along the $m / z(y)$ axis. The longer chain length species are retained slightly more than the shorter chain lengths. The isomers, due to branching of the alkyl chain, elute at slightly different times along the retention time (x) axis, but, of course, at the same $\mathrm{m} / \mathrm{z}$ value. In previous work, we have shown that branched chain isomers elute slightly before straight chain isomers [19]. At least two minor isomeric structures are chromatographically resolved from the main isomer.

Figure 6 shows the contour plot of a commercial EO-PO block copolymer. In other words, the polymer consists of blocks of poly(oxyethylene), also known as polyethylene glycol, ethoxylate or EO, and of poly(oxypropylene), also known as polypropylene gly$\mathrm{col}$, propoxylate or $\mathrm{PO}$. These polymers are used in a wide variety of industrial and consumer product appli- 


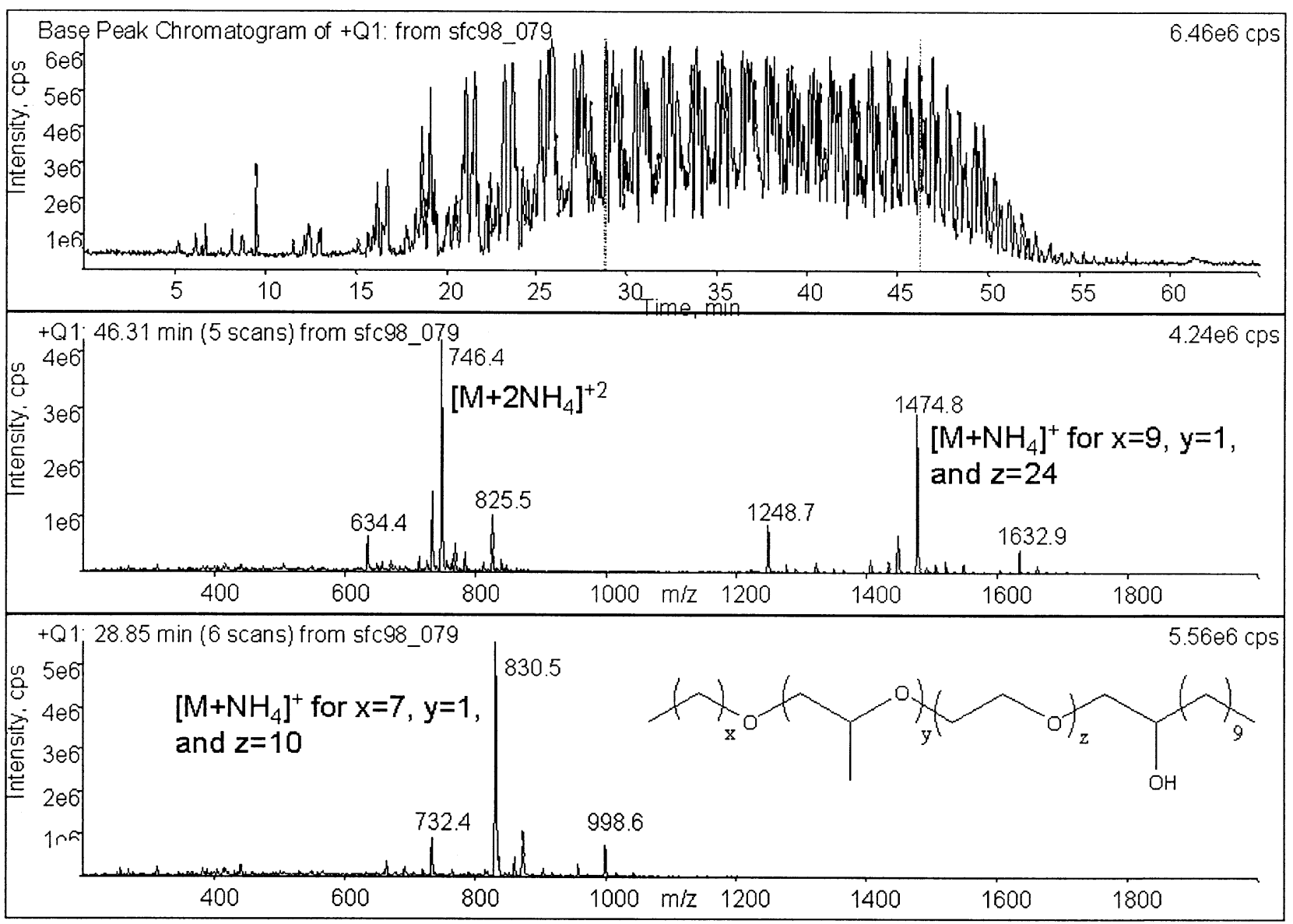

Figure 8. Base peak chromatogram (top) of the di-capped EO-PO polymer shown in Figure 7, and mass spectra of two components of this mixture: The $x=9, y=1$, and $z=24$ component elutes at 46.3 $\min$ (middle), while the $x=7, y=1$, and $z=10$ component elutes at $28.8 \mathrm{~min}$ (bottom). Figures 7 and 8 illustrate the relationship between the contour plot, the base peak chromatogram, and individual mass spectra.

cations, as, for example, emulsifiers, dispersing agents, and wetting agents. The particular polymer featured in Figure 6 is a commercially available suds suppressor. The most prominent series of peaks in the contour plot in Figure 6 is the pure poly(oxypropylene) backbone (a.k.a. poly[propyleneglycol] or PPG) (Figure 6A), indicating that the synthesis of this block co-polymer may have been conducted by ethoxylation of the PO block. Figure 6 illustrates how the patterns produced by the contour plots of the SFC/MS separations can assist in assigning the composition of the polymer. Figure 6 also illustrates how the mass spectrometer and the chromatograph are at least partially orthogonal in their modes of separation. As structures containing only PO groups increase in MW, their $\mathrm{m} / \mathrm{z}$ and their retention time both increase, as indicated in Figure 6A. (Retention of the PO-only structures is primarily driven by nonspecific dispersion forces on the Deltabond Cyano phase.) As EO groups are added to the PO-only structure in the reaction, as shown by arrow " $n$ " in Figure 6, the polarity of the molecule increases, and interactions with the Deltabond Cyano phase increase, contributing to stronger retention and longer retention times. Structures having the same number of oligomeric units, but in which $\mathrm{PO}$ groups are replaced by EO groups, lie along arrow " $\mathrm{m}$ " in Figure 6. As the EO content of the molecule increases relative to the PO content, the MW of the molecule decreases, but its polarity increases. Thus its position drops on the $\mathrm{m} / \mathrm{z}$ scale, but its retention time increases as it is retained more strongly by the relatively polar Deltabond Cyano column. To better illustrate this effect, the region within Figure 6 enclosed with a rectangle has been expanded and some of the peaks have been numbered. These numbers correspond to the peak numbers in Table 1 . The patterns for different types of polymers can be quite distinctive and can not only provide clues on polymer composition, but also on the method used to synthesize the polymer, as has been demonstrated using other mass spectrometric techniques [2-4].

Figure 7 is the contour plot of a slightly more complex EO-PO polymer. The structure of the polymer containing a single capping group on each end of the EO-PO chain, which was deduced from the chromato- 


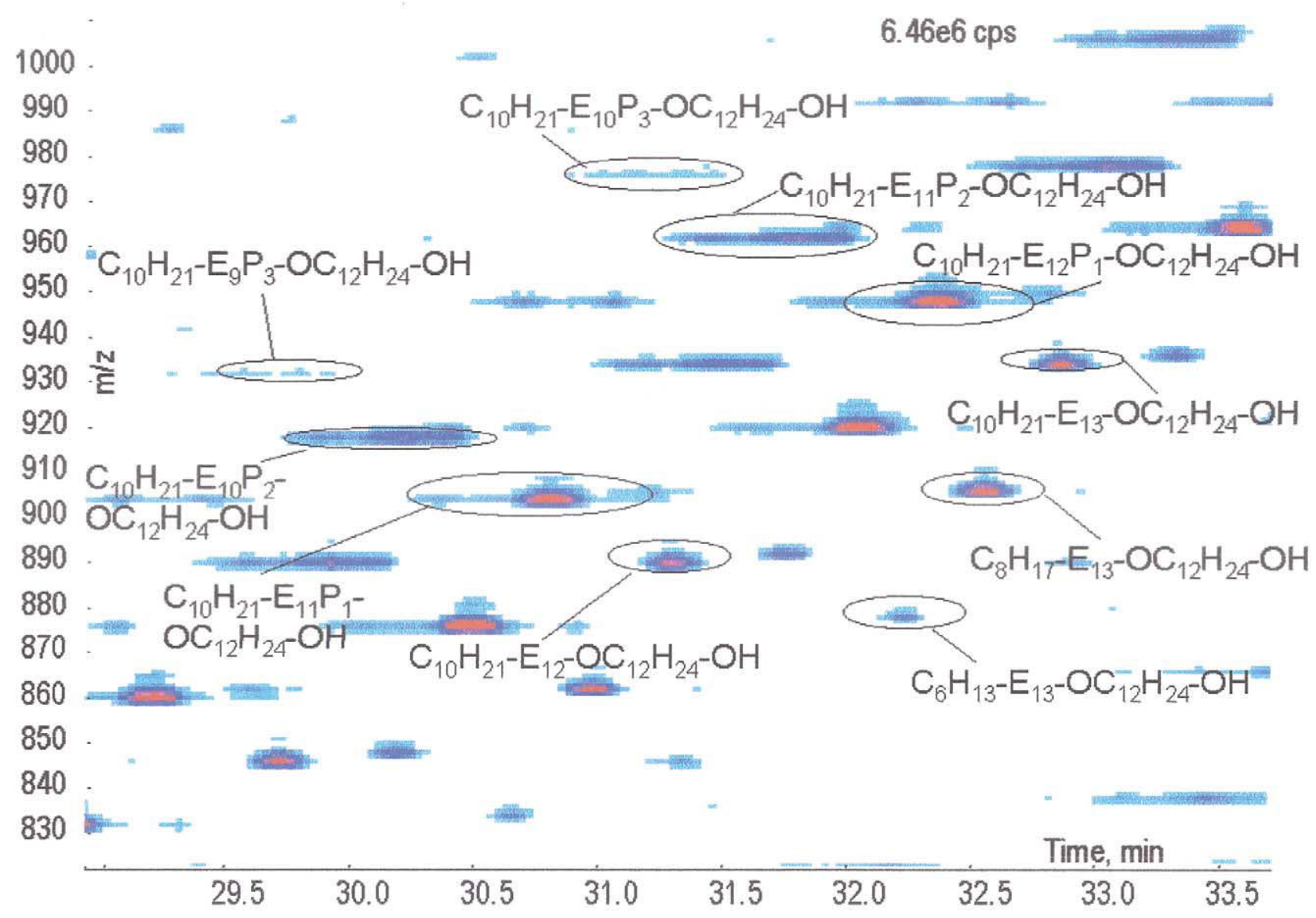

Figure 9. Enlarged portion of Figure 7 showing the di-capped EO-PO polymer region about the elution zone of the polymers containing 12 and 13 ethoxylate groups. The $\mathrm{C} 6, \mathrm{C} 8$, and $\mathrm{C} 10$ alkyl capping groups and the 0-3 propoxylate groups form a distinctive pattern.

graphic pattern, from the $m / z$ values of the ions, and from information about the product, is shown in Figure 7. One end of the alkoxy chain is capped by $\mathrm{C}_{6}, \mathrm{C}_{8}$, and $\mathrm{C}_{10}$ alcohols. The other end of the EO-PO chain is capped with a 12 carbon capping group incorporating a single secondary alcohol functional group. This hydroxyl group can itself react with another identical capping group, yielding dimers, trimers, etc. The singly-capped (no $\mathrm{C}_{12}$ cap, Figure 7B), di-capped (with an alcohol and a monomeric $\mathrm{C}_{12}$, Figure $7 \mathrm{C}$ ), and dicapped (with an alcohol and a dimeric $\mathrm{C}_{12}$, Figure 7D) species correspond to the three major bands of ions ranging in $m / z$ from approximately 500 to nearly 2000 . To better illustrate the relationship between contour plots, base peak chromatograms and mass spectra of individual components, Figure 8 shows the base peak chromatogram for this separation as well as the mass spectra of two components of this complex mixture. The contour plot is a powerful tool in helping the scientist obtain a "top-level" view of the entire mixture. Chromatographic retention patterns, which help in determining structures, are clearly obvious from the contour plot. Figure 9 shows an enlargement of the region of the contour plot containing peaks with a single capping group on each end and with an alkoxylate chain length of 12 and 13 alkoxylate groups. This area clearly reveals the $\mathrm{C}_{6}, \mathrm{C}_{8}$, and $\mathrm{C}_{10}$ capping alcohol pattern and the length of the propoxylate block, ranging from 0 to approximately 3 . The pattern and $m / z$ values shown in Figure 7 indicate that the ethoxylate block ranges in length from approximately 3 through at least 34 ethoxylate units.

\section{Use of Image Analysis for Quantitative Analysis of Polymer Contour Plots}

A typical image derived from an SFC/MS data set, as in Figure 4, has intensities that range over several million units and spatial resolutions that range up to a few thousand by a few thousand pixels! Despite the fact that these images are huge compared to the size of images with which the Optimas image analysis software normally deals, the software, as modified according to the description in the Experimental section, worked smoothly. Once the image is processed to remove background, the analyst can define an arbitrary region in which the peaks are to be detected and integrated (peak intensity multiplied by the base area). Figure 10 shows a data set with background removed and the arbitrary region to be processed outlined. In the next 


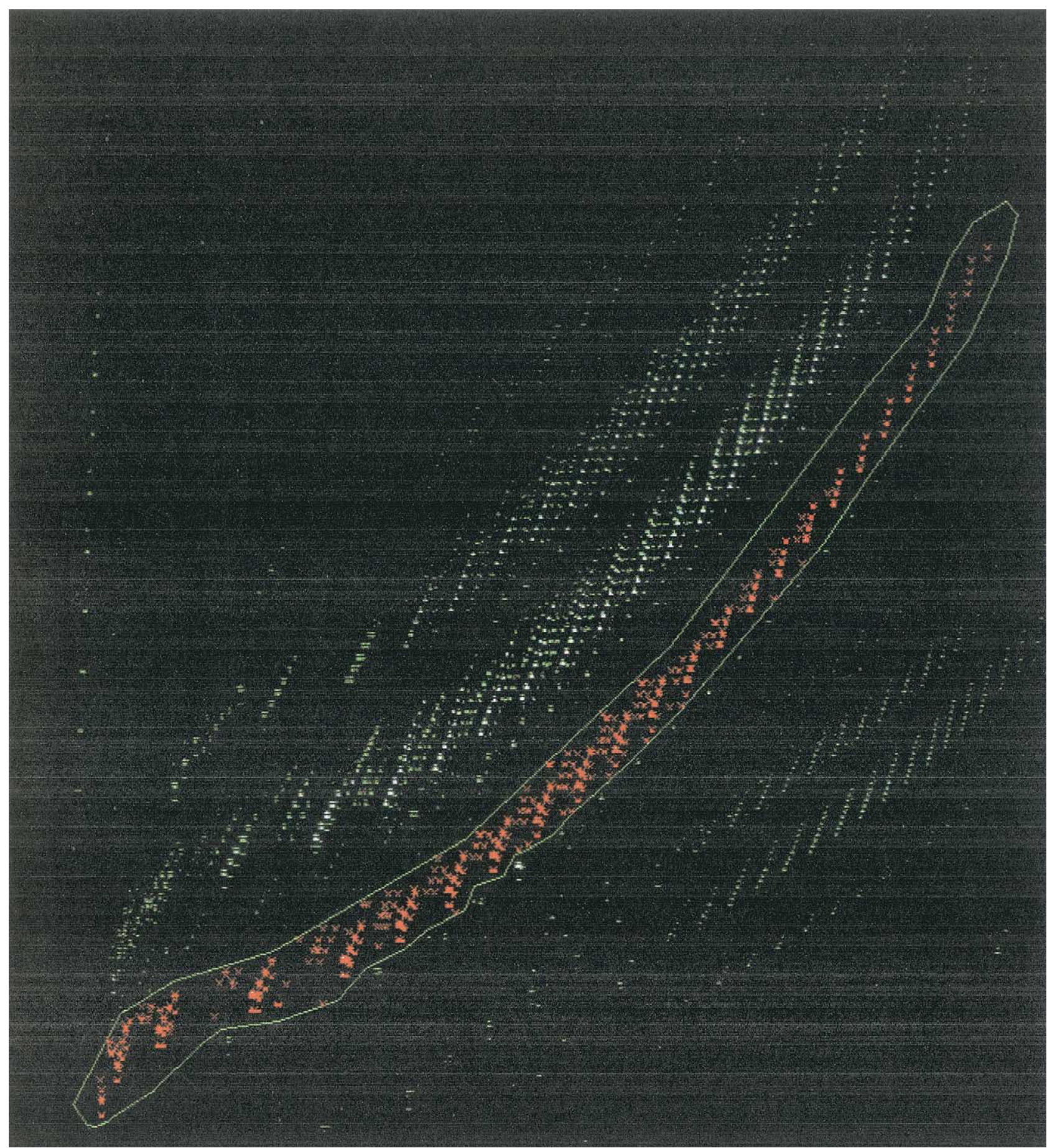

Figure 10. SFC/MS data set similar to that shown in Figure 4 converted to an image file and displayed using the Optimas software. The image has been processed to remove background ions (horizontal lines in Figure 4), a section of the image has been selected by the user for further processing, and the peaks in that section have been detected, as described in the Experimental section. The selected section of the image is outlined and corresponds to the unreacted starting material in this case. The detected peaks are marked with red crosses.

step, the software automatically detects and integrates the peaks. Finally, the software reports the individual peak information (location, intensity, base area), total number of peaks, and the integrated sum of the peak volumes (base area $X$ intensity). These results are then automatically transferred to a Microsoft Excel spreadsheet.

\section{Quantitative Determination of Residual Reactants} Using Image Analysis Tools

While the primary focus of this work was the structural elucidation of low-MW polymers, there are many occasions where quantitative analysis (of residual reactants, 
byproducts, polydispersity measurements, etc.) would be useful. The image analysis tools described in the preceding section have been used to quantify a variety of components in low-MW alkoxylated polymers. For example, they have been used to determine the distribution and quantity of residual reactants. In one experiment, three standard solutions of a commercial $\mathrm{C}_{12}$ ${ }_{15} \mathrm{E}_{12}$ ethoxylated alcohol $\left(\mathrm{C}_{12}, \mathrm{C}_{13}, \mathrm{C}_{14}\right.$, and $\mathrm{C}_{15}$ alcohols ethoxylated to an average ethoxylate chain length of 12) were prepared at concentrations designed to encompass the expected level of residual ethoxylated alcohol. The standards also contained the $\mathrm{C}_{8} \mathrm{E}_{10}$ single component standard described earlier. Once the standards and unknown were characterized by SFC/MS, the total peak volume of the ethoxylated alcohol region of the contour plot was determined using the image analysis tools described above. A calibration curve was constructed from the standards by plotting the ratio of the total AE peak volume to the internal standard peak volume versus the concentration of AE. The correlation coefficient of the curve was 0.9957 . The relative standard deviation of the middle standard $(\mathrm{N}=3)$ was $0.64 \%$ with a total number of peaks above the integration threshold ranging from 440 to 449 . From triplicate analysis of the reaction mixture, the level of residual reactant was determined to be $9.15 \%$.

\section{Conclusions}

Low viscosity mobile phases such as those used in SFC allow the use of long columns for efficient separations of low-MW alkoxylated polymers. When coupled to mass spectrometry, the resulting chromatograms are quite complex, with hundreds of peaks displayed on contour plots of $m / z$ versus retention time versus intensity. These contour plots are powerful tools in structure elucidation. Also, the contour plots can be converted to images, which can be processed using commercial image analysis software with a custom-made user interface. Background is removed from the chromatogram, the user is allowed to define an arbitrary region of interest, chromatographic peaks within this region are detected and integrated, and the results are exported to commercial spreadsheet software. This combination of long column SFC/MS separations with image analysis based data analysis has proven to be a powerful tool in the solution of many problems involving low-MW alkoxylated polymers. Not only has it been useful in quantitating distinct forms of polymers in a complex mixture, but it can also be used in the calculation of parameters such as the average MWs and the polydispersity for these polymer groups.

\section{Acknowledgments}

The authors thank Mark Sivik, Bernie Kleusener, Kevin Garber, and Thomas Mertens for providing many of the samples used in this work, and for useful discussions. They thank Eva Duchoslav and Lyle Burton (PE-Sciex) for the PE-Sciex-to-Matlab data-format conversion script, and for very helpful discussions.

\section{References}

1. Havelka, K. O. Specialty Polymers: Diverse Properties and Applications. ACS Symp. Ser. 2000, 755, 2-10.

2. Shi, S. D. H.; Hendrickson, C. L.; Marshall, A. G.; Simonsick, W. J.; Aaserud, D. J. Identification, Composition, and Asymmetric Formation Mechanism of Glycidyl Methacrylate Butyl Methacrylate Copolymers up to 7000 Da from Electrospray Ionization Ultrahigh-Resolution Fourier Transform Ion Cyclotron Resonance Mass Spectrometry. Anal. Chem. 1998, 70, 3220-3226.

3. Jackson, C. A.; Simonsick, W. J. Application of Mass Spectrometry to the Characterization of Polymers. Cur. Opin. Solid State Mat. Sci. 1997, 2, 661-667.

4. Scrivens, J. H.; Anthony, A. T. Characterization of Synthetic Polymer Systems. Int. J. Mass Spectrom. 2000, 200, 261-276.

5. Pinkston, J. D.; Delaney, T. E.; Bowling, D. J. Characterization of Low Molecular Weight Poly(acrylic acid) Samples by Capillary Supercritical Fluid Chromatography and Capillary Supercritical Fluid Chromatography-Mass Spectrom. J. Microcol. Sep. 1990, 2, 181-187.

6. Carrott, M. J.; Davidson, G. Separation and Characterization of Phenol-Formaldehyde (resol) Prepolymers Using Packed Column Supercritical Fluid Chromatography with APCI Mass Spectrometric Detection. Analyst (Cambridge) 1999, 124, 993997.

7. Yee, R.; Wu, N.; Lee, M. L. Chromatographia (Abstract) 2001, 53, 197-200.

8. Yun, H.; Olesik, S. V.; Marti, E. H. Improvements in Polymer Characterization by Size Exclusion Chromatography and Liquid Chromatography at the Critical Condition by Using Enhanced Fluidity Liquid Mobile Phases with Packed Capillary Columns. Anal. Chem. 1998, 70, 3298-3303.

9. Ute, K. Fractionation of Polymer Homologs with Packed Column Supercritical Fluid Chromatography. In Supercritical Fluid Chromatography with Packed Columns; Anton, K.; Berger, C., Eds.; Marcel Dekker: New York, 1998; pp 349-368.

10. Hoke, S. H. I.; Tomlinson, J. A.; Bolden, R. D.; Morand, K. L.; Pinkston, J. D.; Wehmeyer, K. R. Increasing Bioanalytical Throughput Using pcSFC-MS/MS: 10 Minutes per 96-Well Plate. Anal. Chem. 2001, 73, 3083-3088.

11. Berger, T. A.; Wilson, W. H. Packed Column SupercriticalFluid Chromatography with 220,000 plates. Anal. Chem. 1993, 65, 1451-1455.

12. Yuan, H.; Olesik, S. V. Comparison of Reversed-Phase HPLC Separation Using Carbon Dioxide and Fluoroform for Enhanced Fluidity Liquid Mobile Phases. Anal. Chem. 1998, 70, 1595-1603.

13. Huang, E.; Henion, J.; Covey, T. R. Packed Column Supercritical Fluid Chromatography-Mass Spectrometry and Supercritical Fluid Chromatography-Tandem Mass Spectrometry with Ionization at Atmospheric Pressure. J. Chromatogr. 1990, 511, 367-370.

14. Pinkston, J. D.; Baker, T. R. Modified Ionspray Interface for Supercritical Fluid Chromatography/Mass Spectrometry: Interface Design and Initial Results. Rapid Commun. Mass Spectrom. 1995, 9, 1087-1094.

15. Baker, T. R.; Pinkston, J. D. Development and Application of Packed Column Supercritical Fluid Chromatography/Pneumatically Assisted Electrospray Mass Spectrometry. J. Am. Soc. Mass Spectrom. 1998, 9, 498-509. 
16. Baker, T. R.; Pinkston, J. D. Preliminary Investigation of Supercritical Fluid Assisted Nebulization for Enhanced Response in Electrospray Mass Spectrometry. Analysis 1999, 27, 701-705.

17. Chester, T. L.; Pinkston, J. D. Pressure Regulating Fluid Interface and Phase Behavior Considerations in the Coupling of Packed Column Supercritical Fluid Chromatography with Low Pressure Detectors. J. Chromatogr. 1998, 807, 265-273.
18. Giddings, J. C. Unified Separation Science. John Wiley and Sons: New York, 1991, 105.

19. Smith, C. A.; Pinkston, J. D. unpublished.

20. Dunphy, J. D.; Pessler, D. G.; Morrall, S. W.; Evans, K. A.; Robaugh, D. A.; Fujimoto, G.; Negahban, A. Derivatization LC/MS for the Simultaneous Determination of Fatty Alcohol and Alcohol Ethoxylate Surfactants in Water and Wastewater Samples. Environ. Sci. Technol. 2001, 35, 1223-1230. 